\title{
BESPRECHUNGEN
}




\section{MEDIA GLOBALIZATION IN QUESTION. Ein soziologischer Blick auf medienhistorische Beiträge zur Globalisierungsforschung}

Besprochen von TOBIAS WERRON

Kai Hafez, The Myth of Media Globalization, London (Polity) 2007.

James Schwoch, Global TV. New Media and the Cold War, I946-1969, Champaign (University of Illinois Press) 2009.

Dwayne R. Winseck, Robert M. Pike, Communication and Empire. Media, Markets, and Globalization, I860-1930, Durham (Duke University Press) 2007.

Zur empirischen Medienforschung pflegt die soziologische Globalisierungstheorie eine etwas unentschlossene Beziehung: Einerseits fehlt selten der Hinweis auf die Medien als angeblich zentraler Antriebskraft moderner Globalisierungsprozesse. Anthony Giddens hält sie gar für die eigentliche «Kraft, die das neue Weltzeitalter schafft», ${ }^{1}$ und bei Autoren wie Manuel Castells werden sie zum Kern groß angelegter, die ganze Welt als von Echtzeitmedien verknüpfte «Netzwerkgesellschaft» beschreibender Zeitdiagnosen. Angesichts solch starker Thesen fällt auf, dass historische Analysen der Globalisierungseffekte elektronischer Medien in der globalisierungstheoretischen Literatur selten zu finden sind. Das liegt sicher auch daran, dass viele SoziologInnen und MedientheoretikerInnen dazu neigen, sich für Geschichte - wenn überhaupt - nur als eine Art Kontrastmittel effektvoller Gegenwartsdiagnosen zu interessieren. ${ }^{2}$ Eben diese Neuheitsemphase könnte jedoch mit einem genaueren Blick auf die Mediengeschichte einen wesentlichen Teil ihrer Überzeugungskraft verlieren.

Die drei hier zu besprechenden Bücher decken gemeinsam, bei je unterschiedlichem zeitlichem und thematischem Fokus, den größten Teil der Geschichte der elektronischen Medien seit Mitte des 19. Jahrhunderts ab. Der Zeitraum nach der Einrichtung des Telegrafienetzes in weiten Teilen Europas und Nordamerikas zwischen 1860 und 1930 ist Gegenstand der Studie Communication and Empire von Dwayne R. Winseck und Robert M. Pike, in der sich langjährige Kollaboration und umfassende Archivstudien zu technologischen, ökonomischen und politischen Aspekten globaler Kommunikationsnetze zu einem eindrucksvollen Epochenporträt verbinden. Hauptziel der Autoren ist die Dekonstruktion einer in der Forschung über globale Kommunikation wiederkehrenden 〈Karikatur): Dieser zufolge sei der zwischen 1860 und 1930 entstehende Medienverbund aus Telegrafie, Interkontinentalkabel und Funk, Kommunikationsnetzwerken und globalen Nachrichtenagenturen (wie Reuters, Agence France-Press, Associated Press, Wolff) vor allem von der Rivalität der politischen Supermächte jener Zeit in einem zunächst von Großbritannien, später von den Vereinigten Staaten dominierten «Kampf um die Kontrolle der globalen Kommunikation» angetrieben worden. Winsecks und Pikes These ist, dass genuin ökonomische Dynamiken eine mindestens ebenso große Rolle gespielt haben - was sich u.a. an Kartellen der großen Nachrichtenagenturen, aber auch an Interdependenzen zwischen der Entstehung globaler Märkte und den neuen Informationsnetzwerken ablesen lasse. Sie betonen außerdem, dass Kooperation in vieler Hinsicht im Vergleich zu Kon-

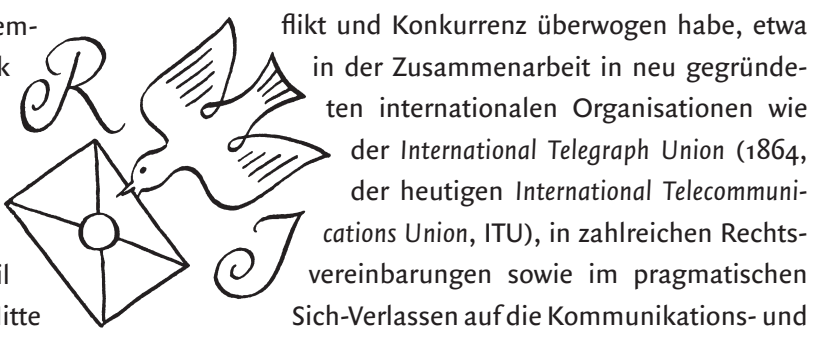


Verwaltungskapazitäten anderer Unternehmen und Staaten (das lediglich im Zuge des Ersten Weltkriegs von ausgeprägteren Antagonismen verdrängt worden sei). Auch die Rolle von idealistischen Reformern wie dem Kanadier Sandford Fleming, dem «Erfinder und Propagandisten der Weltzeit, und anderer sogenannter Internationalisten und Modernisierer findet bei Winseck und Pike mehr Beachtung als üblich. ${ }^{3}$ Diese Akzente fügen sich schließlich auch zu einer Neubewertung dieses «imperialistischen» Zeitalters, das man sich demnach weniger als Aufteilung der Welt in exklusive Herrschaftszonen vorstellen sollte denn als ein "diffuses System", in dem Kooperation und Konkurrenz, Austausch und Konflikt, internationales Recht und Modernisierungs- und Zivilisierungsprojekte auf komplexe Weise zusammengewirkt haben.

Die Fülle der Quellen und Argumente in Winsecks und Pikes beeindruckender Studie kann hier nicht angemessen gewürdigt werden; das Buch bietet en passant auch eine ausgezeichnete Einführung in den Stand empirischer Forschung zu einer Ära, die sich wohl wie keine andere als historische Vergleichsfolie für die heutige Globalisierungsforschung aufdrängt. Ein in seinen theoretischen Konsequenzen noch gar nicht abzusehendes Ergebnis dieser Forschung scheint zu sein, dass sie nicht nur einseitigen ökonomischen oder machtpolitischen Deutungen die Grundlage entzieht, sondern auch die Anfänge dessen, was heute häufig Medialisierung genannt wird, in neuem Licht erscheinen lässt. Denn die Medien treten hier nicht als verselbständigte Nachrichten-, Unterhaltungsund Werbeindustrie auf, als die sie von der kommunikationswissenschaftlichen Forschung meist analysiert werden, sondern als Infrastruktur von Globalisierungsprozessen mit eigener Logik und Temporalität, die sich in dem Untersuchungszeitraum zudem zu einer historisch beispiellosen globalen Integrationsdynamik zusammenschlossen. Dieses Bild tritt hier umso klarer hervor, als Winseck und Pike den idealistischen Zivilisierungsmissionaren jener Zeit mehr Raum geben als üblich. Neben der «imperialistischen» Macht- und der «kapitalistischen» Marktordnung werden so auch die historischen Wurzeln der ontologisch-normativen Weltordnung sichtbar, die heute durch Tausende von Nichtregierungsorganisationen, soziale Bewegungen, investigative Journalisten und andere «rationalisierte Andere» (John W. Meyer) vertreten werden. Dieses Bild könnte sich künftig durch eine Analyse weiterer häufig unter Kultur abgebuchter Bereiche noch erheblich vertiefen und differenzieren lassen, zum
Beispiel indem man nach den Wechselbezügen zwischen Medien und dem im späten 19. Jahrhundert sich formierenden globalen Feld der Religionen ${ }^{4}$ zwischen Medien und Wissenschaft, Medien und Kunst, Medien und Sport etc. fragt und auf dieser Grundlage versucht die Faktoren der Dynamik globaler Integration seit dem 19. Jahrhundert neu zu gewichten.

Bedingungen globaler Integration theoretisch zu gewichten: das heißt mit Blick auf das 20. Jahrhundert nicht zuletzt, die Rolle globaler Konflikte und (Welt-)Kriege zu gewichten, und auch in dieser Hinsicht sind von medienhistorischen Studien wichtige Beiträge zu erwarten. Ein Beispiel für eine Studie dieser Art ist James Schwochs Global TV. New Media and the Cold War, 1946-1969, zu deren Einordnung es sich lohnt, noch einmal zu Winseck und Pike zurückzugehen, in deren Ära ja auch der Erste Weltkrieg und erste Erfolge der drahtlosen Kommunikation fallen: 1903 hatte ein World Radio Congress stattgefunden, 1906 war die Radiotelegraphic Union gegründet und das Prinzip der compulsary intercommunication - das Monopole ausschließen und grenzüberschreitende Kommunikation sichern sollte - beschlossen worden. Diese zunehmende, kooperativ erschlossene und rechtlich abgesicherte kommunikative Vernetzung erstreckte sich u.a. auch auf die karibischen Inseln und Südamerika. All das geschah neben, teils auch in Konkurrenz zu dem bereits bestehenden Kabeltelegrafienetz. Winseck und Pike zeigen nun, dass der Krieg diesen Trend nicht etwa unterbrach, sondern eher noch verstärkte, wie auch umgekehrt die Kriegsführung selbst maßgeblich durch diese neuen Medien geprägt wurde. Beim Ausbau des Funks während des Krieges spielte eine wichtige Rolle, dass grenzüberschreitende Kabelverbindungen leicht unterbrochen werden konnten, zudem weitgehend von Großbritannien kontrolliert waren, so dass die Anreize zum Aufbau kabelloser Netze größer waren denn je, Netze, die dann von allen Kriegsbeteiligten, so zum Beispiel auch von Deutschland in Südamerika, für interne Informations- und externe Propagandazwecke genutzt werden konnten. Die kriegsgesellschaftliche Mobilisierung und Ideologisierung im Ersten Weltkrieg ist folglich keineswegs identisch mit kommunikativer Schließung; vielmehr wurde die effektive globale Vernetzung ihrerseits zum Kriegsprojekt aller Konfliktparteien.

Es ist diese noch in der Kriegssituation selbst sich zeigende Unmöglichkeit und Unerwünschtheit vollständiger Abschließung bei gleichzeitig zunehmendem Beharren 
auf nationalen Grenzen und gleichzeitig zunehmender Fixierung auf nationale Interessen, die auch den Kalten Krieg zu einem medien- und globalisierungshistorisch besonders interessanten Thema macht. Von dieser Phase handelt James Schwochs Buch Global TV. Global war das Fernsehen in Schwochs Augen, weil es sich in einer Zeit zunehmender Globalisierung etablierte, in der politische Fragen auf einer extraterritorialen (transnationalen) Ebene verhandelt wurden, von internationalen Vereinbarungen über das elektromagnetische Spektrum in der ITU, über Unterseekabel, die globale Diffusion von Wissenschaft und Technologie, Atomwaffentests bis hin zu differierenden Visionen von Konsumkultur. Das ist umso bemerkenswerter, als Globalisierung damals ja noch kein gebräuchlicher Begriff war und auch nicht das, was die politischen Akteure im Sinn hatten, denen es, je mehr sich die Lager verfestigten, primär auf eigene Sicherheitsinteressen ankam (kosmopolitische Visionen tauchten hier nur gelegentlich auf - etwa in der Rede von World Citizenship -, gewannen aber wohl in der Spätphase des Ost-West-Konfliktes mehr an Gewicht). Schwoch zieht daraus den plausiblen Schluss, dass Globalisierungsargumente in diesem Fall gleichwohl zutreffend sind, aber gewissermaßen gegen die Absichten der beteiligten Akteure formuliert werden müssten: «Although the word globalization was not in common use by the dramatis personae at that time, globalization can be used retrospectively to help describe, analyze, and understand the actions, ideologies, and discourse of these individuals; that part of the analysis is an analysis transcending the strict limits of intent.» (S.6)

Diese Einsicht ist auch in allgemeiner theoretischer Hinsicht bedeutsam: Vieles, was der Logik des Ost-West-Konfliktes entsprach, folgte einer Logik der Sicherheit und des Wettrüstens, die divergierende Interessen- und Motivlagen unterstellte, diese Divergenz aber in der Regel nicht selbst zum Thema machte und sich den Übergang in die direkte Konfrontation - eben wegen der potenziell zerstörerischen Folgen - meist ersparte. Wie der im Fernsehen inszenierte Wettlauf der Systeme, der hier anschaulich an der Raumfahrt vorgeführt wird, unterstreicht, beruhten diese Divergenzen zudem auf weithin geteilten Modernitäts- und Fortschrittsnormen, die diesen Wettlauf überhaupt erst ermöglichten (worauf bereits in den 196oer Jahren der Soziologe Talcott Parsons aufmerksam machte, der - ein von Schwoch vernachlässigter Punkt - auch auf die Rolle der Weltöffentlichkeit als Adressat in diesem Wettlauf hinwies ${ }^{5}$ ). Soziologisch spricht daher vieles dafür, dass sich der Ost-West-Konflikt im Ganzen überhaupt nicht sinnvoll als Konflikt begreifen lässt, sondern als eine komplexe Mischung aus Kooperations-, Konflikt- und Konkurrenzelementen, deren häufig ungewollte Nebeneffekte erklären, warum diese Phase einer zunehmend scharfen Spaltung der politischen Lager heute zugleich als Phase zunehmender globaler Integration beschrieben werden kann.

Die mit dem Kriterium globale Integration assoziierten Probleme - für globalisierungstheoretisch Interessierte aller Disziplinen von zentraler Bedeutung - berühren auch den Kern des dritten zu besprechenden Buches The Myth of Media Globalization von Kai Hafez. Anders als die beiden anderen ist Hafez' Buch nicht historisch angelegt, gehört aber zu jenen Beiträgen zur Globalisierungsdebatte, die sich erst in dem hier angedeuteten historischen Zusammenhang angemessen bewerten lassen dürften. Der Mythos, von dem Hafez spricht (und dem er im Schlusskapitel immerhin zugesteht, ein «notwendiger Mythos» zu sein), bezieht sich auf neuheitsemphatische Übertreibungen der neueren Globalisierungsforschung, deren «Entmystifizierung" bisher vor allem von Wirtschaftshistorikerlnnen betrieben wurde, die ihren KollegInnen aus Soziologie und Ökonomie den Umfang grenzüberschreitender Handels- und Kapitalströme im späten 19. und frühen 20. Jahrhundert vorgerechnet und gezeigt haben, dass sich vergleichbare Daten im späten 20 . Jahrhundert eher bescheiden ausnehmen. ${ }^{6}$ Hafez wechselt von der Ökonomie zu den Massenmedien und kommt ohne Rückblick auf das 19. Jahrhundert aus, gelangt aber zu einem ähnlich skeptischen Ergebnis, wonach viele globalisierungseuphorische oder -kritische Kollegen und Kolleginnen das wahre Ausmaß der Medienglobalisierung überschätzten. Dabei stützt er sich auf ein theoretisches Modell, das sich für grenzüberschreitende Massenkommunikation zwischen (nationalen) «media systems» interessiert, zwischen direkter (im Ausland produzierter/gesendeter Sendungen) und indirekter (Auslandsberichterstattung durch inländische Medien) grenzüberschreitender Kommunikation unterscheidet und an der so definierten direkten oder indirekten «Konnektivität» das Ausmaß der «Medienglobalisierung» zu messen versucht. Akzeptiert man dieses Modell, kann man Hafez wohl nur zustimmen: Der größte Teil der Medienkommunikation spielt sich in der Tat innerhalb nationaler Grenzen $a b$, und der Konsum ausländischer Medien sowie der Umfang der Auslandsberichterstattung halten sich buchstäblich in engen nationalen Grenzen. 
Anders muss das Urteil jedoch ausfallen, wenn man die Problemkonstruktion selbst in Frage stellt: Wie in der Besprechung der historischen Bücher angedeutet, kann man eine der zentralen Aufgaben der Globalisierungstheorie ja auch darin sehen, die historische Entstehung und Konsolidierung nationaler Grenzen zu erklären, was

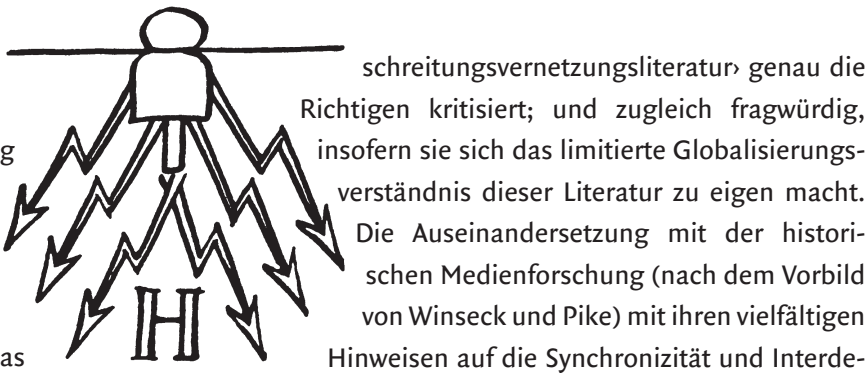
voraussetzt, die grenzüberschreitende Kommunipendenz globaler Beobachtungs- und Vergleichskation nicht selbst zum Definitionsmerkmal der Globalisierung zu erheben. In Globalisierungstheorien wie der neo-institutionalistischen World-Polity-Forschung oder der systemtheoretischen Weltgesellschaftstheorie hat sich deshalb die Ansicht durchgesetzt, dass Globalisierung nicht nur als Vernetzungs-, sondern vor allem als Beobachtungs- und Kommunikationsprozess zu begreifen ist, der über globale Selektionshorizonte oder globales Bewusstsein indirekt auf die Dynamik der Globalisierung zurückwirkt. Kurz: Nicht allein Vernetzung, sondern vor allem weltweit einleuchtende und globale diffundierende Wahrnehmungs- und Vergleichsschemata treiben den Globalisierungsprozess voran. Teilt man diese Ansicht, entfällt eine wichtige Prämisse aller Globalisierungsstudien und -kritiken, die Globalisierung als Phänomen präsentieren, das sich direkt beobachten und zählen lässt, und es hätte auch handgreifliche Konsequenzen für das «Demythifizierungsprojekt» von Hafez (und anderen Autoren): Zu fragen wäre dann weniger, wie viele ausländische Sender konsumiert, wie viel über ausländische Themen berichtet wird, sondern wie dies geschieht - so zum Beispiel in Nachrichtenformaten, die sich weltweit angleichen oder zu Imitations- und Konkurrenzwahrnehmungen unter den Sendern Anlass geben mögen, ohne dass direkte Kontakte oder thematische Bezüge auf ‘das Ausland` sichtbar werden müssten.

Ein stärker auf den Sinngehalt und die Verbreitung von Beobachtungsschemata abstellendes, also phänomenologisches Globalisierungsverständnis führt folglich zu dem Verdacht, dass sich Kritiker wie Hafez die Plausibilität ihrer Argumente teilweise durch einen Globalisierungsbegriff erschleichen, der diese auf grenzüberschreitende Kommunikation, Handelsverbindungen, Kapitalflüsse etc. reduziert und damit eben jene Grenzen reifiziert, deren historische Genese selbst zu den zentralen Themen der Globalisierungsforschung gehören müsste. Darin, könnte man sagen, hat die Kritik am Mythos der Globalisierung ihren eigenen Mythos. Hafez' Kritik ist demnach berechtigt, insofern sie mit den Auswüchsen der «Grenzüberhorizonte auf der einen, nationale Grenzbildung sowie internationale Konflikte und Konkurrenzen auf der anderen Seite mag den Globalisierungstheoretikern helfen, neben dem viel kritisierten methodologischen Nationalismus auch diesen methodologischen Konnektivismus zu korrigieren, der seltener bemerkt wird, aber ebenso problematisch ist.

1 Anthony Giddens, Die große Globalisierungsdebatte, in: Marcus S. Kleiner, Hermann Strasser (Hg.), Globalisierungswelten. Kultur und Gesellschaft in einer entfesselten Welt, Köln (Halem) 2003, 33-47, 36.

2 So aktuell bei Saskia Sassen, Das Paradox des Nationalen. Territorium, Autorität und Rechte im globalen Zeitalter, Frankfurt/M. (Suhrkamp) 2008.

3 Für eine weitere historische Studie, die den Beitrag internationalistischer Idealisten und Organisationen hervorhebt, vgl. Akira Iriye, Global Community. The Role of International Organizations in the Making of the Contemporary World, Berkeley (University of California) 2002, dort mit der Betonung, dass dieser Beitrag gerade in der Zeit zwischen den beiden Weltkriegen - häufig als backlash der Globalisierung (miss-)interpretiert besonders bedeutsam gewesen sei.

4 Zu dieser Neuformierung zu einem Feld von Buchreligionen vgl. nur das anschauliche Religionskapitel in: Christopher Bayly, The Birth of the Modern World 1780-1914. Global Connections and Comparisons, London (Blackwell) 2004.

5 Vgl. Talcott Parsons, Polarization of the World and International Order (1961), in: ders., Sociological Theory and Modern Society, New York (The Free Press) $1967,466-489$.

6 So prominent bei Paul Hirst, Grahame Thompson, Globalization in Question, 2. Aufl., Cambridge (Polity) 2003. 